\title{
Do not assume that no news is good news: test result management and communication in primary care
}

\section{Janice L Kwan, ${ }^{1,2}$ Peter Cram $^{1,2}$}

${ }^{1}$ Department of Medicine, Mount Sinai Hospital, Toronto, Ontario, Canada

${ }^{2}$ Department of Medicine, University of Toronto, Toronto, Ontario, Canada

\section{Correspondence to} Dr Janice L Kwan, Department of Medicine, Mount Sinai Hospital, 427-600 University Avenue, Toronto, Ontario, Canada, M5G 1X5; janice.kwan@utoronto.ca

Accepted 28 July 2015 Published Online First 18 August 2015

\section{Linked}

http://dx.doi.org/10.1136/ bmjqs-2014-003690 - http://dx.doi.org/10.1136/ bmjqs-2014-003712 - http://dx.doi.org/10.1136/ bmjqs-2015-004644

\section{CrossMark}

To cite: Kwan JL, Cram P. BMJ Qual Saf 2015;24: 664-666.
To quote Voltaire by way of Spider-Man, with great power comes great responsibility. This phrase rings especially true for medical trainees on reaching the point in their training when they can order tests without seeking sign off from supervisors. However, they quickly learn that ordering tests is the easy part; managing the resulting data becomes the far more challenging task. Failure to follow-up on test results represents a critical breakdown point in the diagnostic process. ${ }^{1}$ The inherent complexity of test result management, which involves countless steps and handoffs between patients, physicians, nurses and ambulatory office and laboratory staff, is susceptible to failure at multiple provider-level and system-level decision points. Not surprisingly, most primary care physicians express dissatisfaction with their methods for tracking test results, ${ }^{2}{ }^{3}$ and a substantial percentage admit to having no method at all. ${ }^{4}$ Delays in reviewing test results commonly occur, ${ }^{3}$ and approximately one in five errors in the testing process result in patient harm. ${ }^{5}$

Two articles published in BMJ Quality \& Safety remind us just how much work remains to achieve a safe and reliable test result management process. ${ }^{67}$ In the first of these articles, Litchfield and colleagues $^{6}$ conducted a telephone survey of 50 primary care practices in England to evaluate the process of managing and communicating test results. They found that $40 \%$ of practices required patients to call for abnormal results, and that the large majority of practices had no system in place for ensuring return of results from laboratories to practices. In the second of these articles, Litchfield and colleagues $^{7}$ conducted a series of patient and staff focus groups across four primary care practices to understand where unnecessary delays or complete failures in the process could occur. They identified six areas where improvements could be introduced, such as delays in phlebotomy or difficulties in accessing results by telephone. These papers are significant in their own regard, adding much-needed data to a poorly studied problem. More importantly, since virtually all of the research regarding test result management comes from the $\mathrm{USA}^{2-5}$ these papers will help bring international attention to this significant problem.

It is clear that the test result management and communication process is in urgent need of improvement. Proposed solutions have focused on three broad principles: (1) process standardisation with clear assignment of responsibility and accountability for each step across the multidisciplinary team; (2) management tools embedded in the electronic health record (EHR) and (3) improved patient engagement in the process. ${ }^{8}$

The current state for tracking and communicating test results is woefully inconsistent. This appears to hold true, whether focusing on the USA or the UK or at the level of the clinician, small practice or large healthcare system. ${ }^{8}$ There are often no clear protocols for the management of abnormal versus normal versus critical results, timeliness of follow-up and delegation of responsibility. Furthermore, there are rarely clear processes designating responsibility of test result follow-up or communication to patients when the ordering clinician is unavailable. Some healthcare systems, such as the US Veterans Administration (VA) system, do have formal test result policies in place. But, this example represents the exception rather than the rule. Moreover, it is unclear whether policies alone are enough to improve front-line practice. $^{9}$ 
Information technology (IT) solutions have generated considerable enthusiasm. These have most commonly taken the shape of automated test result inboxes or alerts embedded within the EHR, often requiring acknowledgement of notification. However, these IT solutions have generated mixed results ${ }^{10-12}$ and seem unlikely to deliver the silver bullet they were once touted to be. Alert fatigue ${ }^{13} 14$ and system complexity likely share part of the blame. An analysis of alerts for abnormal test results in an integrated EHR at a large VA ambulatory clinic found that just over $10 \%$ of alerts went unacknowledged. ${ }^{11}$ Furthermore, most EHRs currently lack even rudimentary sophistication in test result interpretation, such as the ability to differentiate an elevated serum creatinine in a patient with previously normal renal function (an urgent/emergent result) from an elevated creatinine in a patient on dialysis (a non-urgent result). It appears IT solutions still remain in their infancy.

A growing movement has aimed to put test results directly in the hands of patients. This has been driven in part by financial incentives for meaningful use by the Health Information Technology for Economic and Clinical Health Act of 2009 in the USA and by empowered and engaged patients who want to have greater control over their health. Many EHRs now include 'patient portals', which provide patients with secure and direct access to health information, including test results and clinic notes. They have been promoted as a solution to increase patient activation, while providing an additional layer of safety in the error-prone milieu of test result management. ${ }^{15}$ Patient portals are generally more accepted by patients who are younger, have greater computer literacy and have more trust in the internet. ${ }^{15}$ Moreover, there has been more enthusiasm for portals in patients than physicians. Physician misgivings about portals and direct patient notification of clinically significant test results include concern regarding patient anxiety and confusion about test results, lack of expertise necessary to interpret the results and seeking unreliable information to understand the results. ${ }^{16} \mathrm{~A}$ recent Cochrane review of cellular and mobile technology (or mHealth) for communicating test results found very limited evidence evaluating its utility to date. ${ }^{17}$

As patients increasingly become active partners in healthcare system redesign, it is important to ask what patients want. When it comes to test result communication, one small survey from an ambulatory practice found that almost all patients prefer to receive notification of all test results, both normal and abnormal. Furthermore, patients prefer to have abnormal results accompanied by recommendations for health management changes. ${ }^{18}$ Despite this, fewer than half of providers routinely report all results to patients, ${ }^{9}$ and one-third of physicians do not always notify patients of abnormal test results. ${ }^{4}$ Ideally, patient notification of test results should be timely, reflective of urgency and provide an opportunity for patients to ask questions. ${ }^{19}$ Patient preference appears to depend on two factors-test result 'normalcy' and its 'emotional impact', meaning that a diagnosis of metastatic cancer would likely have a greater emotional impact than would an abnormal vitamin D level. ${ }^{20}$

In a perfect world, all tests would be appropriately ordered, processed and reported in a manner tailored to the individual preference of each patient. However, developing systems to enable such a reality does not seem within our reach at present. This is in part because there will never be a 'one-size-fits-all' approach that will be able to satisfy different individual preferences. ${ }^{21}$ For many, heeding the Agency for Healthcare Research and Quality and one of its top 20 tips to prevent medical errors is very sound advice: 'If you have a test, do not assume that no news is good news. Ask how and when you will get the results'. ${ }^{22}$ Unfortunately, for some patients, sociodemographic and/or clinical factors, such as advanced dementia, limit their ability to self-advocate or access technologies to self-manage their investigations and care. Clearly, the time is now for policymakers to take notice of test result management, an integral part of healthcare quality and patient safety in urgent need of attention.

Competing interests None declared.

Provenance and peer review Commissioned; internally peer-reviewed.

\section{REFERENCES}

1 Gandhi TK, Kachalia A, Thomas EJ, et al. Missed and delayed diagnoses in the ambulatory setting: a study of closed malpractice claims. Ann Intern Med 2006;145:488-96.

2 Murff HJ, Gandhi TK, Karson AK, et al. Primary care physician attitudes concerning follow-up of abnormal test results and ambulatory decision support systems. Int J Med Inform 2003;71:137-49.

3 Poon EG, Gandhi TK, Sequist TD, et al. "I wish I had seen this test result earlier!": dissatisfaction with test result management systems in primary care. Arch Intern Med 2004;164:2223-8.

4 Boohaker EA, Ward RE, Uman JE, et al. Patient notification and follow-up of abnormal test results. A physician survey. Arch Intern Med 1996;156:327-31.

5 Hickner J, Graham DG, Elder NC, et al. Testing process errors and their harms and consequences reported from family medicine practices: a study of the American Academy of Family Physicians National Research Network. Qual Saf Health Care 2008;17:194-200.

6 Litchfield I, Bentham L, Lilford R, et al. Test result communication in primary care-a survey of current practice. BMJ Qual Saf 2015;24:691-9.

7 Litchfield I, Bentham L, Hill A, et al. Routine failures in the process for blood testing and the communication of results to patients in primary care: a qualitative exploration of patient and provider perspectives. BMJ Qual Saf 2015;24:681-90.

8 Callen JL, Westbrook JI, Georgiou A, et al. Failure to follow-up test results for ambulatory patients: a systematic review. J Gen Intern Med 2012;27:1334-48. 
9 Wahls T, Haugen T, Cram P. The continuing problem of missed test results in an integrated health system with an advanced electronic medical record. Jt Comm J Qual Patient Saf 2007;33:485-92.

10 Singh H, Naik AD, Rao R, et al. Reducing diagnostic errors through effective communication: harnessing the power of information technology. J Gen Intern Med 2008;23:489-94.

11 Singh H, Thomas EJ, Sittig DF, et al. Notification of abnormal lab test results in an electronic medical record: do any safety concerns remain? Am J Med 2010;123:238-44.

12 Murphy DR, Reis B, Sittig DF, et al. Notifications received by primary care practitioners in electronic health records: a taxonomy and time analysis. Am J Med 2012;125:209.e1-7.

13 Nanji KC, Slight SP, Seger DL, et al. Overrides of medication-related clinical decision support alerts in outpatients. J Am Med Inform Assoc 2014;21:487-91.

14 Isaac T, Weissman JS, Davis RB, et al. Overrides of medication alerts in ambulatory care. Arch Intern Med 2009;169:305-11.

15 Goldzweig CL, Orshansky G, Paige NM, et al. Electronic patient portals: evidence on health outcomes, satisfaction, efficiency, and attitudes a systematic review. Ann Intern Med 2013;159:677-87.
16 Giardina TD, Callen J, Georgiou A, et al. Releasing test results directly to patients: A multisite survey of physician perspectives. Patient Educ Couns 2015;98:788-96.

17 Gurol-Urganci I, de Jongh T, Vodopivec-Jamsek V, et al. Mobile phone messaging for communicating results of medical investigations. Cochrane Database Syst Rev 2012; 6:CD007456.

18 Meza JP, Webster DS. Patient preferences for laboratory test results notification. Am J Manag Care 2000;6:1297-300.

19 Choudhry A, Hong J, Chong K, et al. Patients' preferences for biopsy result notification in an era of electronic messaging methods. JAMA Dermatol 2015;151:513-21.

20 Shultz SK, Wu R, Matelski JJ, et al. Patient preferences for test result notification. J Gen Intern Med Published Online First: 6 May 2015. doi:10.1007/s11606-015-3344-0

21 Crotty BH, Walker J, Dierks M, et al. Information sharing preferences of older patients and their families. JAMA Intern Med Published Online First: 6 Jul 2015. doi:10.1001/ jamainternmed.2015.2903

22 Agency for Healthcare Research and Quality. 20 Tips to Help Prevent Medical Errors. December 2014. http://www.ahrq.gov/ patients-consumers/care-planning/errors/20tips/index.html (accessed 18 Jul 2015). 